\title{
Modelos de predicción del rendimiento académico de los estudiantes de medicina en el ciclo básico y preclínico. Un estudio longitudinal
}

\author{
Denisse Zúñigala, Beltrán Mena², Rose O livaa, \\ N uria Pedrals ${ }^{3 a}$, 0 slando Padilla4b, Marcela Bitran'c. \\ Modeling the academic performance \\ of medical students in basic sciences \\ and pre-clinical courses: \\ A longitudinal study
}

Background: The study of predictors of academic performance is relevant for medical education. Most studies of academic performance use global ratings as outcome measure, and do not evaluate the influence of the assessment methods. Aim: To model by multivariate analysis, the academic performance of medical considering, besides academic and demographic variables, the methods used to assess students' learning and their preferred modes of information processing. Material and methods: Two hundred seventy two students admitted to the medical school of the Pontificia Universidad Católica de Chile from 2000 to 2003. Six groups of variables were studied to model the students' performance in five basic science courses (Anatomy, Biology, Calculus, Chemistry and Physics) and two pre-clinical courses (Integrated Medical Clinic I and II). The assessment methods examined were multiple choice question tests, Objective Structured Clinical Examination and tutor appraisal. Results: The results of the university admission tests (high school grades, mathematics and biology tests), the assessment methods used, the curricular year and previous application to medical school, were predictors of academic performance. The information processing modes influenced academic performance, but only in interaction with other variables. Perception (abstract or concrete) interacted with the assessment methods, and information use (active or reflexive), with sex. The correlation between the real and predicted grades was 0.7. Conclusions: In addition to the academic results obtained prior to university entrance, the methods of assessment used in the university and the information processing modes influence the academic performance of medical students in basic and preclinical courses (Rev Méd Chile 2009; 137: 1291-300).

(Key words: Learning; Students, medical; Task performance and analysis)

\footnotetext{
Recibido el 10 de marzo, 2009. Aceptado el 7 de septiembre, 2009.

${ }^{1}$ Centro de Educación Médica, Escuela de Medicina, Pontificia Universidad Católica de Chile. ${ }^{2}$ Oficina Editorial, Escuela de Medicina, Pontificia Universidad Católica de Chile. ${ }^{3}$ Dirección General Estudiantil, Pontificia Universidad Católica de Chile. ${ }^{4}$ Departamento de Salud Pública, Escuela de Medicina, Pontificia Universidad Católica de Chile.

aPsicóloga

${ }^{b}$ Estadístico

${ }^{\mathrm{c}} \mathrm{PhD}$ en Farmacología
}

Correspondencia a: Denisse Zúñiga Parada. Centro de Educación Médica, Escuela de Medicina, Pontificia Universidad Católica de Chile. Lira 40, edificio Medicina $6^{\circ}$ piso. Fono: 354-3512. Fax: 633-1457. E mail: dzunigpa@uc.cl 
$\mathrm{E}^{\mathrm{I}}$ 1 estudio de predictores del rendimiento académico es relevante en educación médica ${ }^{1,2}$. Investigadores de ciencias de la salud y de otras disciplinas han intentado identificar de qué forma, variables tradicionales de ingreso a la universidad, notas de secundaria, así como variables demográficas y estilos de aprendizaje, contribuyen al desempeño académico de pregrado ${ }^{1,3-10}$. Existen estudios que han centrado el análisis en algunas variables $^{4,5,11,12}$; mientras que otros han intentado responder a la pregunta a través del contraste de variables y diseño de modelos de predicción más complejos ${ }^{13-18}$

En medicina se han desarrollado modelos predictivos que incorporan variables distintas a las pruebas de ingreso a la universidad. Edad, sexo, resultados en entrevistas de admisión, experiencia académica previa, experiencias con temas de salud previa, personalidad y motivación se relacionan con rendimiento académico ${ }^{13,17}$. En otras carreras de pregrado, los estilos de aprendizaje ${ }^{15}$, las creencias de los estudiantes ${ }^{18}$ y variables de contexto $^{16}$, se relacionan con rendimiento académico.

En general, los estudios de rendimiento académico suelen utilizar indicadores globales de evaluación (promedio general por período o promedio final de cursos) sin considerar las calificaciones según los métodos de evaluación empleados. Duff y cols ${ }^{15}$ sugieren la necesidad de avanzar en estudios de predicción del rendimiento que incluyan los distintos tipos de evaluación.

Desde el modelo constructivista y de la psicología del desarrollo, se ha demostrado que en el aprendizaje intervienen varios factores. Las características del objeto a aprender, las variables personales (cognitivas, biológicas y socio-afectivas) del que aprende y las variables contextuales (metodología enseñanza, institucional, etc.) ${ }^{19-21}$. Además, las funciones cognitivas que se alcanzan en la adolescencia permanecen hasta la adultez y lo que varía es el curso que toman, producto de las experiencias a las que se está expuesto ${ }^{21}$.

El supuesto a la base de este estudio es que el rendimiento (como expresión del aprendizaje) no sólo puede ser analizado desde las calificaciones sino que debe considerar otras variables, como por ejemplo, el contexto en que estos aprendizajes son evaluados (métodos de evaluación, año de la carrera, tipo de asignatura) o la experiencia (intereses, actitivades) y estrategias cognitivas que el aprendiz activa en su desarrollo (estilos de aprendizaje).

El propósito del presente estudio es modelar el rendimiento académico de 4 cohortes de estudiantes de medicina de la Pontificia Universidad Católica de Chile, con el objetivo de responder a dos preguntas: ¿Existen otras variables, además del puntaje de ingreso, que predicen el rendimiento académico de los estudiantes?, ¿influyen los métodos de evaluación y el procesamiento de la información en el rendimiento académico de los estudiantes en el ciclo básico y preclínico?

\section{Materiales y MÉTODOS}

Población. Doscientos setenta y dos estudiantes admitidos en el primer año de la carrera de medicina de la Pontificia Universidad Católica de Chile (PUC) entre los años 2000 y 2003 (edad al ingreso: 18,6 $\pm 1,1$ años; sexo: 44\% mujeres).

Modos de procesamiento de la información. Los modos de procesamiento de la información se evaluaron a través del Inventario de Estilos de Aprendizajes de Kolb (IEA, versión en español) que se aplicó en $1^{\mathrm{er}}$ y $3^{\mathrm{er}}$ año (Aplicación 1 y 2, respectivamente), previo consentimiento informado de los participantes.

El IEA ${ }^{22,23}$, basado en la Teoría de Aprendizaje Experiencial, identifica las preferencias de cada individuo por uno de los dos modos de adquirir información: Conceptualización Abstracta (CA), o bien, Experiencia Concreta (EC); y por uno de los dos modos de utilizarla: Observación Reflexiva (OR) o Experimentación Activa (EA).

Los aprendices 'Abstractos' (CA $>$ EC) se caracterizan por su facilidad para identificar conceptos y relaciones, y distinguir patrones generales de información. Mientras, los 'Concretos' (EC>CA) se distinguen por usar la experiencia directa y trabajar en equipo. Desde el modo de utilizar la información, los aprendices 'Activos' (EA>OR) tienden a probar distintas alternativas sin realizar un análisis previo exhaustivo y a utilizar el conocimiento para resolver problemas. En cambio, los 'Reflexivos' (OR>EA) examinan acuciosamente las alternativas antes de actuar. 
Tipo de experiencia previa al ingreso a la universidad. Al momento de aplicar el IEA, los estudiantes completaron una Ficha de Antecedentes sobre el tipo de experiencia académica (preuniversitario, postulación previa a medicina, estudios universitarios) y no académica (trabajo voluntario, trabajo remunerado y actividades recreativas, sean deportivas, religiosas o artísticas) realizadas antes de ingresar a medicina en la PUC.

Pruebas de ingreso a la universidad. Se analizaron los resultados de las pruebas de ingreso exigidos para postular a medicina en la PUC entre 2000 y 2003. La Prueba de Aptitud Verbal (PAV), la Prueba de Aptitud Matemática (PAM), la Prueba de Conocimientos Específicos de Biología (PCEBio) y la Prueba de Aptitud de Historia y Geografía (PAHG); junto con las Notas de Enseñanza Media (NEM) y el Puntaje de Selección que se obtiene de la ponderación que aportan las pruebas y el NEM (20\% PAV, 25\% PAM, 25\% PCEBio, $10 \%$ PAHG y $20 \%$ NEM).

Antecedentes curriculares. Año en que se dicta el curso. En $1^{\mathrm{er}}$ año se dictan las asignaturas básicas de Anatomía, Cálculo, Química y Física; y en $2^{\circ}$ año se dicta Biología. En $3^{\text {er }}$ y $4^{\circ}$ año se imparten los cursos Integrado de Clínicas Médicas I y II, respectivamente ${ }^{24}$.

\section{Métodos de evaluación}

a. La Prueba de Múltiple Elección (ME) evalúa conocimientos teóricos. Se analizaron las notas de Anatomía, Cálculo, Química, Física y Biología; así como también, el rendimiento obtenido con este método en los cursos Integrado de Clínicas Médicas I y II.

b. La Nota de Tutor (NT), en ambos cursos PreClínicos, tiene como propósito evaluar la experiencia clínica con pacientes. En general, mide la capacidad de comunicarse con los pacientes, la calidad del anamnesis y del examen físico, la capacidad de establecer hipótesis y diagnóstico, y la interpretación de exámenes de laboratorio. El Integrado de Clínicas Médicas II incluye, además, la proposición de un plan de tratamiento.

c. La Evaluación Clínica Objetiva Estructurada (ECOE) del Integrado de Clínicas Médicas I está basada en situaciones clínicas que evalúan la interacción con un paciente (real o simulado), el reconocimiento de radiografías u otros exámenes básicos y el enfrentamiento a determinados problemas clínicos. Adicionalmente, el ECOE del Integrado de Clínicas Médicas II, evalúa conocimientos de fisiopatología y clíni$\mathrm{ca}$, el empleo de herramientas diagnósticas y de comunicación médico-paciente.

Las notas obtenidas con los distintos métodos de evaluación, incluyendo el ECOE que tradicionalmente se calcula como porcentaje de logro, se expresarán en escala de 1 a 7 para hacer posible el modelaje estadístico.

Todas las variables descritas se ilustran en la Figura 1.

Estadística. Como parte de los análisis bivariados se realizaron tests chi-cuadrado para la comparación de frecuencias, cálculo de coeficientes de correlación de Pearson y análisis de varianza (ANOVA).

El análisis multivariado se basó en la aplicación de modelos mixtos, una clase más general que los modelos de regresión lineal, para explicar o modelar las notas de los estudiantes a partir de un conjunto de variables predictoras. Los modelos mixtos también son una generalización del ANOVA y en este sentido permiten concluir desde el punto de vista estadístico si un factor tiene impacto sobre la variable respuesta (las notas de los estudiantes) una vez ajustado por las otras variables. Los modelos mixtos también generalizan los modelos de medidas repetidas y en nuestro caso permiten la modelación de la correlación entre las diferentes notas de un mismo estudiante.

El nombre de modelos mixtos proviene de la consideración de algunas variables como efectos fijos y otras como efectos aleatorios. Si los niveles de una variable son los únicos posibles (sexo) o los únicos sobre los que interesa hacer inferencia (por ejemplo, $1^{\mathrm{er}}$ año, $2^{\mathrm{o}}$ año de carrera), entonces estos niveles se consideran como efecto fijo y entran en el modelo lineal como una constante (coeficiente). Si en cambio, al observar un grupo de personas lo que interesa es hacer inferencia a toda la población, no sólo a los individuos observados, entonces en el modelo lineal el 'efecto persona' no entra como una constante sino como una realización de una variable aleatoria y 


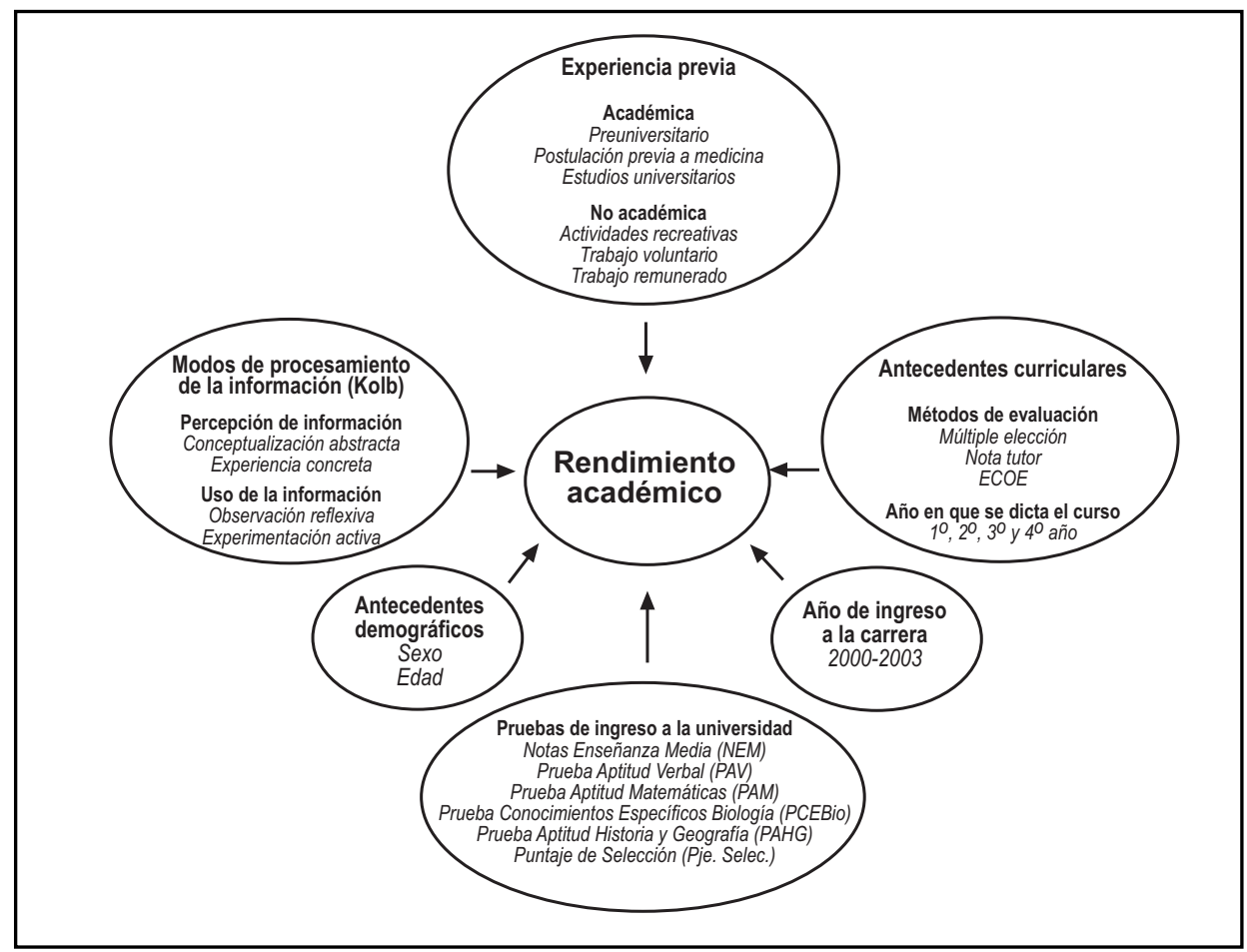

Figura 1. Variables analizadas en el modelo de predicción del rendimiento académico de los estudiantes de medicina (cohortes 2000-2003).

eso da lugar a parámetros de varianza adicionales, cuya estimación no es trivial, pero para la cual ya se han implementado algoritmos de estimación disponibles en software como el usado en este trabajo.

Los análisis estadísticos se llevaron a cabo en el software SPSS ${ }^{\circledR}$ versión 16.0.2. Se consideraron significativos valores de $\mathrm{p}<0,05$.

\section{Resultados}

Tipo de experiencia previo al ingreso a medicina. La Tabla 1 indica el número y porcentaje de estudiantes que tuvo algún tipo de experiencia académica o no académica antes de ingresar a medicina PUC.

Modos de procesamiento de la información. La Tabla 2 muestra la distribución de estudiantes según el modo de percibir y usar la información, en la aplicación 1 (al ingreso) y la aplicación 2 (al tercer año). La distribución de los estudiantes para ambos modos es similar en la Aplicación 2 (valor $\mathrm{p}>0,10$ ).

En la Tabla 3 se observan el puntaje promedio y desviación estándar obtenidos por los estudiantes en el IEA en ambas aplicaciones, organizados según los modos de percepción de la información (Tabla 3a) y los modos de uso de la información (Tabla 3b). Los resultados obtenidos en los modos de percepción y uso de la información de la Aplicación 1 son similares a los de la Aplicación 2.

Rendimiento en pruebas de ingreso a la universidad. La Tabla 4 muestra la media y desviación estándar del puntaje obtenido por todos los estudiantes en las distintas pruebas de ingreso a la universidad.

Rendimiento en asignaturas básicas y preclínicas. La Tabla 5 muestra el desempeño de los estudiantes en las asignaturas básicas y preclínicas según 
Tabla 1. Tipo de experiencia académica y no académica de los estudiantes previo al ingreso a medicina (cohortes 2000-2003)

\begin{tabular}{|lcc|}
\hline & $\mathbf{n}$ (n=272) & \% \\
\hline Experiencia académica & & \\
$\quad$ Preuniversitario & 230 & 85 \\
$\quad$ Postulación previa a medicina & 72 & 27 \\
$\quad$ Estudios universitarios & 36 & 13 \\
Experiencia no académica & 245 & 90 \\
$\quad$ Actividades recreativas & 158 & 58 \\
Trabajo voluntario & 81 & 30 \\
Trabajo remunerado & & \\
\hline
\end{tabular}

Tabla 2. D istribución de los estudiantes de medicina (cohortes 2000-2003) según modos de procesamiento de la información (IEA, Kolb 1981) en la Aplicación 1 y Aplicación 2

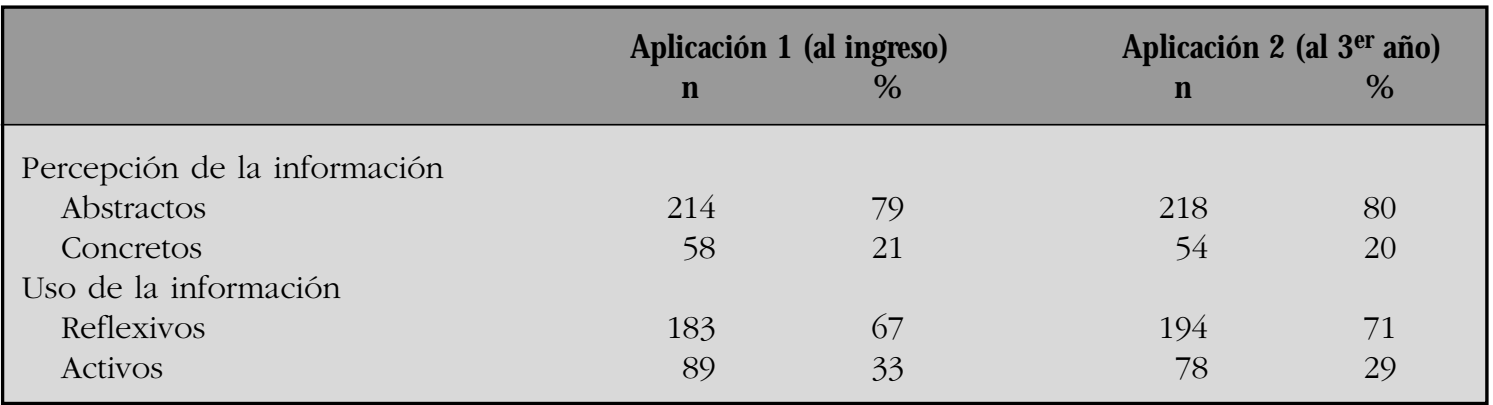

Tabla 3. M edia y desviación estándar de los modos de procesamiento de la información (IEA, Kolb 1981) obtenidas por los estudiantes de medicina (cohortes 2000-2003) en la Aplicación 1 y Aplicación 2

\begin{tabular}{|c|c|c|c|c|c|c|}
\hline & \multicolumn{6}{|c|}{ 3a. Percepción de la información } \\
\hline & \multicolumn{2}{|c|}{ CA } & \multicolumn{2}{|c|}{ EC } & \multicolumn{2}{|c|}{ D iferencia C A - EC } \\
\hline & Aplicación 1 & Aplicación 2 & Aplicación 1 & Aplicación 2 & Aplicación 1 & Aplicación 2 \\
\hline Abstractos & $38,3 \pm 5,4$ & $39,3 \pm 6,5$ & $21,0 \pm 4,8$ & $20,1 \pm 5,7$ & $17,3 \pm 0,6$ & $19,2 \pm 0,8$ \\
\hline \multirow[t]{4}{*}{ Concretos } & $25,1 \pm 5,2$ & $27,1 \pm 7,6$ & $33,0 \pm 7,0$ & $31,2 \pm 8,7$ & $-7,8 \pm-1,8$ & $-4,2 \pm-1,1$ \\
\hline & \multicolumn{6}{|c|}{ 3b. Uso de la información } \\
\hline & \multicolumn{2}{|c|}{ EA } & \multicolumn{2}{|c|}{ OR } & \multicolumn{2}{|c|}{ D iferencia EA - OR } \\
\hline & Aplicación 1 & Aplicación 2 & Aplicación 1 & Aplicación 2 & Aplicación 1 & Aplicación 2 \\
\hline Activos & $37,2 \pm 4,5$ & $36,3 \pm 5,9$ & $24,7 \pm 4,5$ & $24,9 \pm 5,5$ & $12,5 \pm 0$ & $11,4 \pm 0,4$ \\
\hline Reflexivos & $27,0 \pm 4,2$ & $27,8 \pm 6,2$ & $33,5 \pm 5,6$ & $33,0 \pm 5,7$ & $-6,5 \pm-1,4$ & $-5,2 \pm-0,5$ \\
\hline
\end{tabular}

CA: Conceptualización Abstracta; EC: Experiencia Concreta; EA: Experimentación Activa; OR: Observación Reflexiva. 
Tabla 4. Media y desviación estándar de las pruebas de ingreso a la universidad obtenidas por los estudiantes de medicina (cohortes 2000-2003)

\begin{tabular}{|lc|}
\hline & Media \pm DE \\
\hline Puntaje de selección & $772 \pm 12$ \\
Notas de Enseñanza Media (NEM) & $763 \pm 38$ \\
Prueba de Aptitud Verbal & $762 \pm 30$ \\
Prueba de Aptitud Matemática (PAM) & $790 \pm 24$ \\
Prueba de Conocimientos Específicos de Biología (PCEBio) & $769 \pm 42$ \\
Prueba de Aptitud de Historia y Geografía (PAHG) & $770 \pm 31$ \\
\hline
\end{tabular}

Tabla 5. M edia y desviación estándar de las asignaturas básicas y preclínicas según métodos de evaluación obtenidas por los estudiantes de medicina (cohorte 2000-2003)

\begin{tabular}{|lccc|}
\hline & $\begin{array}{c}\text { Múltiple elección } \\
\text { (media } \pm \mathbf{D E} \text { ) }\end{array}$ & $\begin{array}{c}\text { Nota tutor } \\
\text { (media } \pm \mathbf{D E} \text { ) }\end{array}$ & $\begin{array}{c}\text { ECOE } \\
\text { (media } \pm \mathbf{D E} \text { ) }\end{array}$ \\
\hline $\begin{array}{c}\text { Asignaturas básicas } \\
\text { Anatomía }\end{array}$ & $5,5 \pm 0,5$ & n.a. & n.a. \\
Biología & $5,6 \pm 0,6$ & n.a. & n.a. \\
Física & $5,7 \pm 0,6$ & n.a. & n.a. \\
Cálculo & $5,4 \pm 0,7$ & n.a. & n.a. \\
Química & $5,1 \pm 0,9$ & n.a. & n.a. \\
Asignaturas preclínicas & & & $5,5 \pm 0,4$ \\
Integrado Clínicas Médicas I & $5,9 \pm 0,5$ & $6,5 \pm 0,3$ & $5,3 \pm 0,6$ \\
Integrado Clínicas Médicas II & $5,8 \pm 0,5$ & $6,5 \pm 0,2$ & \\
\hline
\end{tabular}

ECOE: Evaluación clínica objetiva estructurada; n.a.: no aplica el método de evaluación.

los distintos modos de evaluación que lo componen. Las asignaturas básicas sólo son evaluadas con tests de Múltiple Elección (ME).

Al comparar el rendimiento del Integrado de Clínicas Médicas I según el modo de evaluación, observamos que la Nota de Tutor (NT) tiene el promedio más alto y la Evaluación Clínica Objetiva y Estructurada (ECOE), el más bajo. Esto es similar en el Integrado Clínicas Médicas II. Por otro lado, el promedio del Integrado Clínico I y II en cada uno de los métodos de evaluación es similar.

Matriz de correlaciones de Pearson. La Tabla 6 presenta la matriz de correlaciones entre el rendimiento obtenido por los estudiantes en las asigna- turas básicas y preclínicas con las pruebas de ingreso y los modos de procesamiento de la información.

Se encontraron correlaciones significativas entre el rendimiento de casi todas las asignaturas básicas y el Puntaje de Selección y NEM. Las demás pruebas se correlacionan sólo con algunas asignaturas básicas. No existen correlaciones entre las notas de las asignaturas básicas y los modos de procesamiento de la información, a excepción del modo de percepción "Abstracto" (CA) y el rendimiento en Cálculo.

En los Integrados I y II se encontraron algunas correlaciones entre el rendimiento (en los distintos tipos de evaluación) y algunas pruebas de ingreso a la universidad. NT y ECOE del Integrado de Clínicas Médicas I y II correlacionan con el 
Tabla 6. M atriz de correlaciones entre el rendimiento obtenido en las asignaturas básicas y preclínicas, en la pruebas de ingreso a la universidad y los modos de procesamiento de la información (IEA, Kolb, 1981) de los estudiantes de medicina (cohortes 2000-2003)

\begin{tabular}{|c|c|c|c|c|c|c|c|c|c|c|c|c|}
\hline & & \multicolumn{5}{|c|}{ Asignaturas básicas } & \multicolumn{6}{|c|}{ Asignaturas preclínicas } \\
\hline & & \multirow[b]{2}{*}{ Cálculo } & \multirow[b]{2}{*}{ Q uímica } & \multirow[b]{2}{*}{ Anatomía } & \multirow[b]{2}{*}{ Física } & \multirow[b]{2}{*}{ Biología } & \multicolumn{3}{|c|}{$\begin{array}{l}\text { Integrado Clínicas } \\
\text { Médicas I }\end{array}$} & \multicolumn{3}{|c|}{$\begin{array}{l}\text { Integrado Clínicas } \\
\text { M édicas II }\end{array}$} \\
\hline & & & & & & & ME & NT & ECOE & ME & NT & ECOE \\
\hline \multirow{6}{*}{$\begin{array}{l}\text { Prueba de } \\
\text { ingreso a la } \\
\text { universidad }\end{array}$} & Pje. Selec. &, $341^{* *}$ & ,301*** & 222 & & , 184** & &, $210^{* *}$ & ,213*** &, $129^{*}$ & ,241** &, $153^{*}$ \\
\hline & NEM &, $268^{* *}$ &, $167^{* *}$ &, $137^{*}$ &, $140^{*}$ &, $239^{* *}$ &, $174^{*}$ &, $197^{* *}$ & &, $138^{*}$ &, $141^{*}$ & \\
\hline & PCEBio & &, $132^{*}$ &, $185^{* * *}$ & & & &, $125^{*}$ & ,128* & & & \\
\hline & PAHG & & &, $152^{*}$ & & ,135* & & & & & ,129* & \\
\hline & PAM & ,298** &, $158^{* * *}$ & & & & & & & & & \\
\hline & PAV & & &,$- 169^{* *}$ &,$- 179 * *$ & & & & & & & \\
\hline Modos de & $\mathrm{CA}$ & ,119* & & & & & ,148* & & & & ,156* & \\
\hline procesamiento & EC & & & & & & & & & & & \\
\hline de la & EA & & & & & &,$- 147^{*}$ & & & &,$- 142^{*}$ & \\
\hline información & OR & & & & & & & & &,$- 130^{*}$ & & \\
\hline
\end{tabular}

ME: Múltiple elección; NT: Nota tutor; ECOE: Evaluación clínica objetiva estructurada; Pje. Selec.: Puntaje selección; NEM: Notas enseñanza media; PAM: Prueba aptitud matemática; PAV: Prueba aptitud verbal; PAHG: Prueba aptitud historia y geografía; PCEBio: Prueba conocimientos específicos biología; CA: Conceptualización Abstracta; EC: Experiencia Concreta; EA: Experimentación Activa; OR: Observación Reflexiva. **p $<0,01$. *p $<0,05$.

Puntaje de Selección. ME del Integrado II también correlaciona con el Puntaje de Selección. Por otro lado, ME y NT de ambos cursos preclínicos correlacionan con NEM. NT y ECOE del Integrado I correlacionan con la PCEBio. Finalmente, se observa una correlación entre la NT del Integrado II y la PAHG. No se encontraron correlaciones entre las asignaturas preclínicas y la PAM ni la PAV. En todos los casos descritos, las correlaciones son positivas.

Se observan algunas correlaciones entre métodos de evaluación y los modos de procesamiento de la información. En el Integrado I, ME correlaciona positivamente con el modo de percepción CA y negativamente con el modo de uso "Activo" (EA). En cambio, en el Integrado II se encontró, por un lado, una correlación negativa entre ME y el modo de uso "reflexivo" por otro, NT correlacionó positivamente con el modo de percepción CA y negativamente con el modo de uso EA. En ninguno de los cursos preclínicos se encontraron correlaciones entre el ECOE y los modos de procesamiento de la información (Tabla 6).
Modelos predictores del rendimiento académico. La Tabla 7 muestra las variables relevantes del Modelo 1 (estudiantes como efecto fijo) y el Modelo 2 (estudiantes como efecto aleatorio).

De acuerdo al Modelo 1, las variables: trabajo voluntario y remunerado, postulación previa a medicina, año de ingreso a medicina, puntajes de ingreso en NEM, PAM, PCEBio y PAHG; año en que se realiza el curso y métodos de evaluación fueron predictores del rendimiento académico en el ciclo básico y preclínico. El procesamiento de la información influyó en el rendimiento, en interacción con otras variables: la percepción con los métodos de evaluación y el uso con el sexo.

El Modelo 2 mantiene la mayoría de los efectos observados en el Modelo 1, a excepción de trabajo remunerado y voluntario, la PAHG y el año de ingreso. De acuerdo a las medidas de bondad de ajuste observadas, como el criterio de Akaike, ambos modelos tienen valores semejantes (Tabla 7). Sin embargo, el Modelo 2 ajusta mejor los datos, esto se puede apreciar en el valor de correlación observado -en la Tabla $7(\mathrm{r}=0,759)$ 


\section{Tabla 7. Nivel de significancia de las variables predictoras del rendimiento académico de los estudiantes de medicina (cohortes 2000-2003) analizadas en los M odelos 1 y 2}

\begin{tabular}{|c|c|c|}
\hline Variables seleccionadas & $\begin{array}{l}\text { Modelo } 1 \\
\text { efecto fijo }\end{array}$ & $\begin{array}{l}\text { Modelo } 2 \\
\text { efecto aleatorio }\end{array}$ \\
\hline Trabajo voluntario & $*$ & \\
\hline Trabajo remunerado & * & \\
\hline Postulación previa a medicina & **** & $* * *$ \\
\hline Notas enseñanza media (NEM) & $* * *$ & $* * *$ \\
\hline Prueba aptitud matemática (PAM) & **** & **** \\
\hline Prueba conocimientos específicos biología (PCEBio) & **** & $* * *$ \\
\hline Prueba aptitud historia y geografía (PAHG) & **** & \\
\hline Año de ingreso a la carrera & $* * *$ & \\
\hline Año en que se dicta el curso & $* * * *$ & $* * *$ \\
\hline Método de evaluación & $* * *$ & $* * *$ \\
\hline Método de evaluación x modo percepción de la información & $* *$ & $* *$ \\
\hline Sexo x modo de uso de la información & $*$ & $*$ \\
\hline Criterio de bondad de ajuste Akaike (AIC) & 5541,7 & 5117,8 \\
\hline Correlación pronosticados vs observados, $\mathrm{r}=$ & 0,625 & 0,759 \\
\hline
\end{tabular}

${ }^{* * * *} \mathrm{p}<0,001 . * * \mathrm{p}<0,01 . * \mathrm{p}<0,05$.

que se obtiene al estudiar la asociación entre los valores observados (data) y los pronosticados (predicción) (Figura 2).

Las variables predictoras comunes en ambos modelos influyen en el rendimiento académico de los estudiantes de la siguiente manera: 1) Experiencia académica previa: los estudiantes que postulan por primera vez a medicina tienen mejor rendimiento que aquellos que lo han intentado antes; 2) Pruebas de ingreso a la universidad: estudiantes con altos puntajes en NEM, PAM y PCEBio obtienen mejor desempeño académico; 3) Antecedentes curriculares: por un lado, los estudiantes obtienen mejores calificaciones en las asignaturas que se imparten en $3^{\mathrm{er}}$ y $4^{\mathrm{O}}$ año (preclínicas) que en las de $1^{\circ}$ y $2^{\mathrm{O}}$ año (básicas). Por otro, los estudiantes rinden mejor en NT y ME que en ECOE; 4) Interacción entre los modos de procesamiento de la información con otras variables: por una parte, los modos de percepción de la información interactúan con los métodos de evaluación: en ME los estudiantes 'Abstractos' rinden mejor que los 'Concretos', mientras que en el ECOE a los 'Concretos' les va mejor que a los
'Abstractos'. Por otra parte, los modos de uso de la información interactúan con sexo. Los 'hombres Activos' obtienen mejores notas que las 'mujeres Activas', mientras que las 'mujeres Reflexivas' rinden mejor que los 'hombres Reflexivos'.

\section{DisCUSIÓN}

¿Existen otras variables, además del puntaje de ingreso, que sean predictoras del rendimiento académico de los estudiantes de medicina? ¿Influyen los métodos de evaluación y el procesamiento de la información en el rendimiento académico de los estudiantes en el ciclo básico y preclínico?

Variables curriculares e individuales, además de las pruebas de ingreso, resultaron ser predictoras del rendimiento académico básico y preclínico de los estudiantes de medicina de 4 cohortes. Los predictores incluyen los métodos de evaluación y los modos de procesamiento de la información.

De un total de 29 variables modeladas, resultaron predictoras: la postulación previa a medicina, 3 de las pruebas de ingreso a la universidad (las 


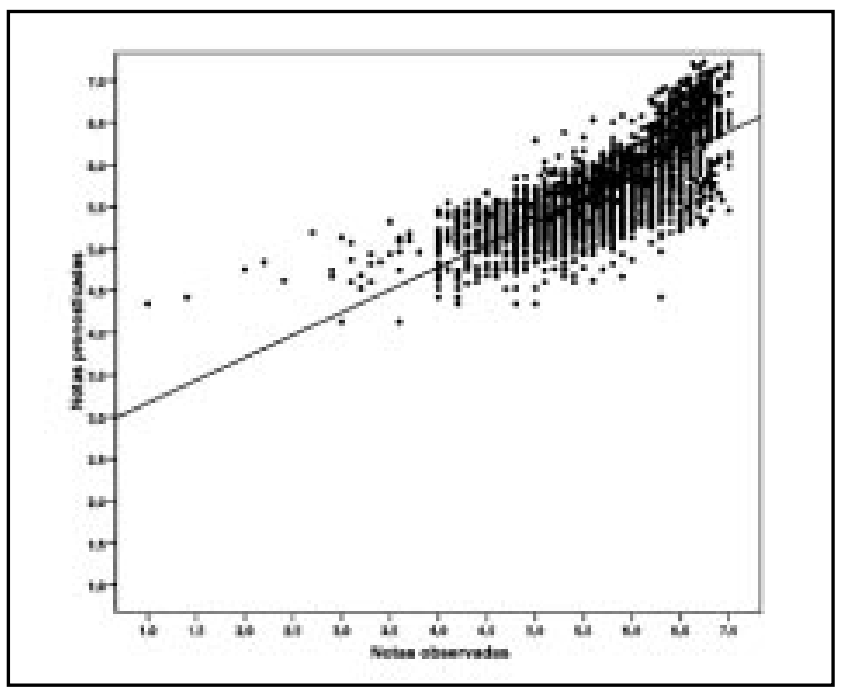

Figura 2. Gráfico de correlación de las notas pronosticadas y observadas según el modelo 2.

notas de Enseñanza Media, el puntaje de la Prueba de Aptitud Matemática y de Conocimientos Específicos de Biología) y los antecedentes curriculares (año en que se dicta el curso y métodos de evaluación). El procesamiento de la información influyó en el rendimiento, en interacción con otras variables: el modo de percepción de información (abstracto o concreto) con los métodos de evaluación, y el uso de la información (activo o reflexivo), con sexo.

Si bien se propusieron dos modelos, se consideraron como predictoras relevantes las variables del modelo 2. Este modelo posee varias ventajas: considera a los estudiantes como efecto aleatorio lo que permite extrapolar los resultados a otras cohortes de estudiantes no consideradas en el estudio. Además, es un modelo de predicción más parsimonioso, proporciona un mejor ajuste de los datos y un menor número de variables.

Las revisiones realizadas por Ferguson $^{1}$ y Regerh $^{2}$ advierten la escasez de modelos predictivos, y entonces, la necesidad de analizar integralmente los diversos factores que inciden en el rendimiento académico de los estudiantes de medicina de pregrado.

Nuestro estudio, desde este punto de vista, permite contribuir en dos aspectos. Primero, si bien la literatura informa respecto del poder predictivo de las variables individuales (cognitivas y no cognitivas) ${ }^{1-18}$, es nueva la información respecto de las variables contextuales (curriculares) -tales como el año curricular y los métodos de evaluación-, que dependen de la institución que forma a los estudiantes. Segundo, los resultados sobre los estilos de aprendizaje y su relación con el rendimiento son poco concluyentes ${ }^{1,3-6,11}$. Un hallazgo interesante del estudio es la forma particular en que las estrategias de aprendizaje (modos de procesamiento de la información) resultan ser predictores de rendimiento académico en interacción con otras variables individuales (sexo) y curriculares (métodos de evaluación).

Consistente con investigaciones previas, nuestros hallazgos confirman el poder predictor que tienen las pruebas de ingreso $0^{1,7,8,10}$ y la experiencia previa en el rendimiento de pregrado $10,11,13-18$. Consideración tienen las variables trabajo comunitario y remunerado que no se mantuvieron en el modelo de efecto aleatorio. Son interesantes desde el punto de vista del impacto que puede llegar a tener la participación de los estudiantes en otras actividades que no sean académicas, y que el tipo de trabajo al que se dedican los estudiantes influyen en sus resultados académicos. 
Creemos que los resultados del presente estudio deben interpretarse con precaución. Por una parte, es importante completar el modelaje del rendimiento en el ciclo terminal de la carrera, y examinar cuán generalizable es este modelo de predicción en otras realidades universitarias.

\section{REFERENCIAS}

1. Ferguson E, James D, Madeley L. Factors associated with success in medical school: systematic review of the literature. BMJ 2002; 324: 952-7.

2. Regerh G. Trends in Medical Education Research. Acad Med 2004; 79: 939-47.

3. SAndmire D, VRoman K, SAnders R. The influence of learning styles on collaborative performances of allied health students in a clinical exercise. J Allied Health 2000; 29: 143-9.

4. Lynch T, Woelfl N, Steele D, Hanssen C. Learning style influences student examination performance. Am J Surg 1998; 176: 62-6.

5. Martin I, Stark P, Jolly B. Benefiting from clinical experience: the influence of learning style and clinical experience on performance in an undergraduate objective structured clinical examination. Med Educ 2000; 34: 530-4.

6. McManus I, Richards P, Winder B, Sproton K. Clinical experience, performance in final examinations, and learning style in medical students: prospective study. BMJ 1998; 316: 345-50.

7. Bastías G, Villarroel L, Zúñiga P, Marshall G, Velasco N, Mena B. Desempeño académico de los estudiantes de medicina: ¿Un resultado predecible? Rev Méd Chile 2000; 128: 671-8.

8. Meleca B. Tradicional predictors of academic performance in a medical school's independent study program. Acad Med 1995; 70: 59-63.

9. McGanney M, Ganoo S. Noncognitive factors and performance predictions. Acad Med 1995; 70: 1-2.

10. Bitran M, Wright aC, Zúñiga D, Mena B, Velasco N, Moreno R. Mejoría en el desempeño académico de estudiantes de medicina en tiempos de reforma curricular. Rev Méd Chile 2002; 130: 437-45.

11. Bitran M, Lafuente M, Zúñiga D, Viviani P, Mena B. ¿Influyen las características psicológicas y los estilos de aprendizaje en el rendimiento académico de los estudiantes de medicina? Un estudio retrospectivo. Rev Méd Chile 2004; 132: 1127-36.

12. Lievens F, Coetsier P, De Fruyt F, De Maeseneer J. Medical students' personality characteristics and
Limitaciones. Una limitación de este modelo predictivo es que, aun cuando tiene un valor de correlación alto, no es tan útil para predecir las notas bajas. Otra limitación es la homogeneidad del rendimiento académico de los estudiantes.

academic performance: a five-factor model perspective. Med Educ 2002; 36: 1050-6.

13. Madigan V. Predicting Hospital Care Students' FirstYear Academic Performance. Prehosp Emerg Care 2006; 10: 81-8.

14. Stoddard H. Crossing the bridge to diversity: sucess in medical school for students who are underrepresented in medicine. A PRESSENTATION. University of Nebraskalincoln 2005. Disponible en: http://proquest.umi.com/ pqdweb?index $=138 \mathrm{did}=921028461 \&$ SrchMode $=1 \&$ sid $=3 \&$ Fmt $=2 \&$ VInst $=$ PROD $\&$ VType $=$ PQD $\&$ RQT $=309 \&$ VName $=$ PQD\&TS=1233329586\&clientld=52738 [Consultado el 28 enero 2009].

15. Duff A, Boyle E, Dunleavy K, Ferguson J. The relationship between personality, approach to learning and academic performance. Pers Individ Dif 2004; 36: 1907-20.

16. Fenollar P, Román S, Cuestas P. University students' academic performance: an integrative conceptual framework and empirical analysis. Br J Edu Psychol 2007; 77: 873-91.

17. Hoschl M, Kozeny J. Predicting academic performance of medical students: The First Three years. Am J Psychiatry 1997; 154: 87-92.

18. Phan H. Predicting changes in epistemological beliefs, reflective thinking and learning styles: A longitudinal study. Br J Edu Psychol 2008; 78: 75-93.

19. Rosas R, SEBAstián C. Piaget, Vigotski y Maturana. Constructivismo a tres voces. Buenos Aires: Aique. 2008.

20. Vigotsky L. Obras escogidas (I y II). Barcelona: Fuente Labrada. 1993.

21. Undurraga C. ¿Cómo aprenden los adultos? Santiago: Ediciones Universidad Católica de Chile. 2004.

22. KolB D. Inventario de los estilos de aprendizaje: Inventario autoevaluativo y su interpretación. Boston: Training Resources Group, Hay/Mc Ber. 1981.

23. Kolb D. Experiential Learning: Experience as the Source of Learning and Development. New Jersey: Prentice Hall, PTR. 1984.

24. Sánchez I, Riquelme A, Moreno R, Mena B, Dagnino J, GreBE G. Revitalising medical education: the school of medicine at the Pontificia Universidad Católica de Chile. Clin Teach 2008; 5: 57-61. 